
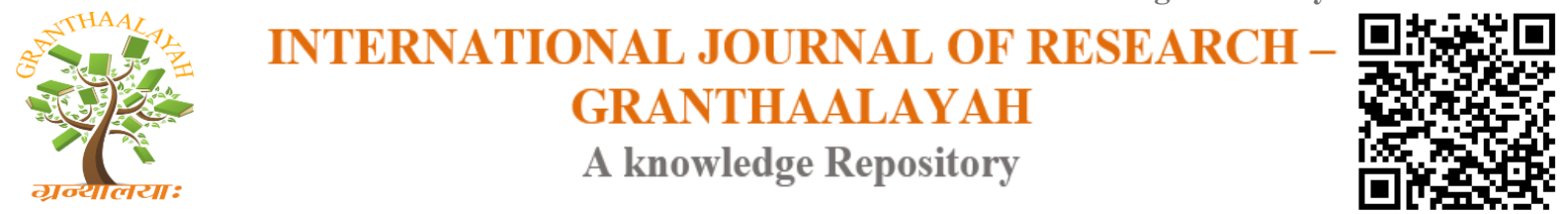

Science

\title{
A SURVEY ON OPTIMAL IPV4 TO IPV6 TRANSITION TECHNIQUES
}

\author{
Aparna Sivaprakash ${ }^{*}$, S. Kayalvizhi ${ }^{2}$ \\ ${ }^{* 1,2}$ Computer Science and Engineering, Easwari Engineering College, Chennai, INDIA
}

\begin{abstract}
The number of global internet users has been growing exponentially, thereby requiring a much larger number of unique IP addresses for all the connected networking devices. The prevalent IP version 4 is not able to meet the current requirement for IP addresses and to meet the future IP address requirement, a new version IPv6 has been introduced since 1999. However IPv6 is not backward compatible with IPv4.since it is not possible to migrate all the networking devices to IPv6 in a single day, IPv4 and IPv6 are going to be used in parallel for some time. Here we aim to provide a Literature Survey which showcases the various different techniques to implement IPv4 to IPv6 transition and figure out the most optimal method to increase the network performance.
\end{abstract}

Keywords:

Tunneling, Translation, Dual Stack, IPv4, IPv6.

Cite This Article: Aparna Sivaprakash, and S. Kayalvizhi, "A SURVEY ON OPTIMAL IPV4 TO IPV6 TRANSITION TECHNIQUES" International Journal of Research - Granthaalayah, Vol. 4, No. 4 (2016): 90-96.

\section{INTRODUCTION}

Networking can be defined as the process of linking various hardware and software devices together by using data communications processes. The Internet Protocol (IP) within a network is responsible for addressing hosts and routing datagrams from a source device to the destination device through various IP networks.

A. IPv4 and IPv6

Internet Protocol version 4 (IPv4) is the fourth revision in the development of the Internet Protocol (IP) and is one of the most widely used internet layer protocols. IPv4 is a connectionless protocol used for packet-switched Link Layer networks (e.g., Ethernet). It operates on a best effort delivery and makes use of 32-bit addressing. However IPv4 addresses have been exhausted over time due to the increasing number of devices using the internet. The IP address space of all devices is managed by the Internet Assigned Numbers Authority (IANA) globally, and by five regional Internet registries (RIR). With IANA's exhaustion on 31 January 2011, some parts of 
world have already exhausted the remaining addresses. The shortage of IPv4 addresses was the major factor that resulted in creating and adopting several new technologies, including IPv6 (Internet Protocol Version 6).

\begin{tabular}{|c|c|c|c|c|}
\hline VER & HL & TOS & \multicolumn{2}{c|}{ TOTAL LENGTH } \\
\hline \multicolumn{2}{|c|}{ IDENTIFICATION } & FLAG & FRAGMENT OFFSET \\
\hline TTL & PROTOCOL & HEADER CHECKSUM \\
\hline \multicolumn{3}{|c|}{ SOURCE ADDRESS } \\
\hline \multicolumn{3}{|c|}{ DESTINATION ADDRESS } \\
\hline \multicolumn{3}{|c|}{ OPTIONS } \\
\hline
\end{tabular}

Figure 1: IPv4 Header Format

IPv6 makes use of 128-bit addresses, and so the new address space supports $2^{128}$ addresses. The 128-bit addresses are subdivided into 8 groups. These 8 groups are further divided into 4 digit hexadecimal numbers separated by colons. The resulting representation is called colonhexadecimal. Although the new protocol IPv6 is in place currently, a large number of present devices were built for IPv4. Hence it is mandatory that the two protocols communicate with each other. In order for the coexistence to work perfectly, we need some kind of transition method to relocate the currently being used IPv4 addresses to IPv6 addresses, and for this purpose we make use of two techniques called Tunneling and Dual Stack.

\begin{tabular}{|l|l|l|}
\hline VER & TRAFFIC CLASS & \multicolumn{2}{|c|}{ FLOW LABEL } \\
\hline PAYLOAD LENGTH & NEXT HEADER & HOP LINIT \\
\hline \multirow{3}{*}{ SOLRCE ADDRESS } \\
\hline \multicolumn{3}{|c|}{ DESTINATION ADDRESS } \\
\hline
\end{tabular}

Figure 2: IPv6 Header Format

\section{B. $\quad$ Tunneling and Dual Stack}

A Tunnel can be defined as an Internet Protocol (IP) communications channel between any two networks. In a Tunnel, gateways are used to create the endpoints at the borders between the source network and the destination networks. Thereby, the tunnel endpoints become native routers that establish a standard route between the source and destination networks. Any data 
packets travelling through these end-points are stripped from their basic frame format headers and trailers and then converted into a native IP format and injected into the IP stack of endpoints. In addition to this any other protocol encapsulations used during transit, such as IPsec or Transport Layer Security, is also removed from the packet.

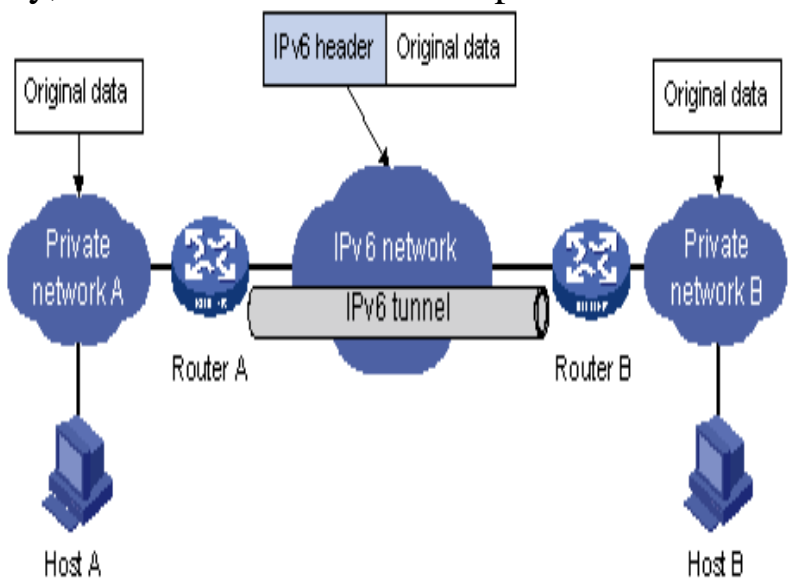

Figure 3: Tunneling Mechanism

Dual Stack Transition Mechanism (DSTM) on the other hand, is a mechanism based on the usage of IPv4-over-IPv6 tunnels to allow interoperability between newly deployed IPv6 networks and existing IPv4 networks. DSTM is most suitable for IPv6 dominant environments where hosts still need to exchange information with legacy IPv4 hosts or applications. In a dualstack environment, all devices can communicate with not only IPv6 systems, but also with IPv4 systems. Applications can choose whether to use IPv6 or IPv4, by selecting the correct address based on the type of IP traffic and particular requirements of the communication.

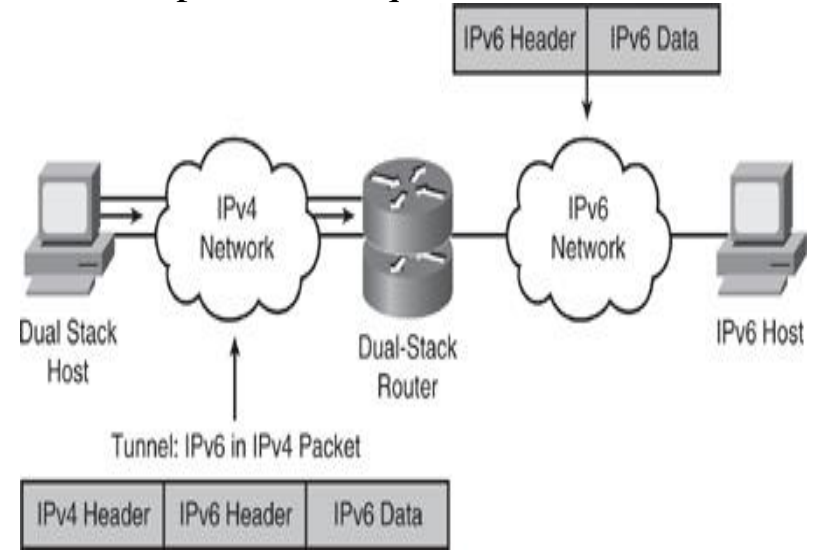

Figure 4: Dual Stack Mechanism

While dealing with both IPv4 and IPv6, although a comparative study has been done between Dual Stack and Tunneling, protocol specific performance evaluation is yet to be determined. A case by case study is necessary, as all the protocols may not adapt equally across devices and platforms. An assessment of all possible combinations of the migration techniques and routing protocols is yet to be performed. Performance of the system after implementing the routing protocols has to be analysed. 


\section{RELATED WORKS}

Tachapat Taesawat and Suwat Pattarmalai (2014) [8] proposed a mechanism for performance comparison of Dual Stack and Tunneling mechanisms in data transferring of internet protocol version 6 transitions. Network models for both techniques are created in GNS3 with fixed assigned data transferring route. Moreover, UDP data streaming and TCP file downloading are used with various rate and file sizes. Finally, the simulation results show that dual stack has more throughputs and less delay jitter in the UDP results. It also gives shorter time to finish file downloading in TCP results. The issue with this system however is that although comparative study between the migration techniques have been performed, the examination of all possible combinations of RIPng and OSPFv3 with the migration techniques has not yet been studied.

Yong Cui, Jiang Dong, Peng Wu and Jianping Wu, Chris Metz, Yiu L. Lee, and Alain Durand (2013) [1] have developed a tunnel-based framework that solves the transition problems in backbone and access networks with different tunneling mechanisms. IPv6 transition presents many challenges to the Internet community, and various solutions have been proposed, including dual stack, tunneling, and translation. Tunneling supports like-to-like IP connectivity across an unlike network, whereas translation supports like-to-unlike IP interconnectivity. Tunneling operations include encapsulation, de capsulation, and tunnel endpoint signalling, with no upper layer operation required. The authors have applied IPv6 Rapid Deployment to provide IPv6 connectivity in IPv4 access networks. The drawback of this process however is the low flexibility and an overwhelming management load for ISPs.

Peng Wu, Yong Cui, Jianping Wu, Jiangchuan Liu, and Chris Metz (2013) [2] have conducted a state of the art survey for Transition from IPv4 to IPv6. It considers the basic problems and difficulties in IPv4-IPv6 transition. It then surveys the two mainstream tunneling and translation mechanisms. Moreover, the authors also look into the characteristics and transition requirements of practical ISP networks, and propose the usage and deployment strategies of the transition mechanisms in both backbone and edge networks. Both Translation and Tunneling can be further subdivided in the following categories respectively; Stateless Translation, Stateful Translation, Host side translation, Tunnel Mesh Mechanisms, and Host-to-host Tunnel Mechanisms. The transition techniques however are found to be facing challenges and require further research efforts. For translation techniques, the most critical issue is the lack of feasible, stateful IPv4 $\rightarrow$ IPv6 translation mechanisms.

Krishna Chakraborty, Nitul Dutta, S R Biradar (2009) [5] proposed a comparative study of the behaviour of IPv4-only network with that of Dual Stack Transition Mechanism (DSTM) under various types of traffic patterns. In the proposed DSTM enabled network architecture, the hosts in IPv4 network initiates connection with hosts in the IPv4 network over an integrated IPv4/IPv6 network. This research concludes that in spite of imposing extra delay to the network, the DSTM is significant for the following two reasons. Firstly, a transition mechanism is required for the smooth interoperation of both the protocols and secondly, DSTM has proved to have several features of Tunneling and Dual-Stack approach which can be thought of as an intermediate of these two transition mechanisms. However The RA (Real Audio) traffic consumes maximum time as it is real-time application and hence the payload will also end up being more. It is also seen that there is a significant increase in the delay for RA traffic. 
Yuk-Nam Law, Man-Chiu Lai, Wee Lum Tan and Wing Cheong Lau (2008) [6] have proposed a comprehensive empirical measurement of the IPv6 network performance from an end user's perspective. They quantify the performance differences of using IPv6 vs. IPv4, in terms of various network metrics like network connectivity, hop count, RTT, throughput, operating systems dependencies as well as the address configuration latency. In general the measurements have shown that the IPv6 network is able to provide stable network connectivity for IPv6 endhosts. On the other hand, there is still considerable room for improvement in terms of reducing the IPv6 path RTT through the deployment of more IPv6 nodes. The results also show the need for an improvement in the IPv6 performance of Windows-based clients, as compared to Unixbased clients. This is necessary in order to reduce the dependence of IPv6 performance on the type of operating systems used by the IPv6 end-hosts.

Dipti Chauhan and Sanjay Sharma (2015) [3] have proposed a method for enhancing the efficiency of IPv6 Tunnelling mechanism by using header compression over IPv6 header. This approach is implemented over IPv6 tunneling mechanism and around 40 bytes of IPv6 header have been compressed up to 6 bytes. At the sender side an additional parameter is used. If the parameter is 0 , then normal Tunneling mechanism is used. If it is 1 , the compressed header method is used. Simulation results of the data show that using this approach provides better network deliverables in terms of throughput, average end-to-end delay, Jitter, and Packet delivery ratio. However the results have been obtained only for small networks with lesser number of nodes and their performance in large term wired and wireless networks has yet to be seen.

Priya Bali (2015) [7] provides a detailed comprehensive review on IPv4-to-IPv6 transition and co-existence strategies along with comparison of their performances to show how these transition strategies affects network behaviour. Three methods, Tunneling, Dual Stack and Translation are used for the comparison. Both manual transition techniques as well as automatic IPv6 transition are reviewed. Based on various interviews and research it can be concluded that native DualStack is the technology that companies should consider for their deployment. Apart from this the next best transition technology to deploy in the network is NAT64. Some companies plan on using this technique and then move to native IPv6 when all devices are ready to support it. If the devices are not ready, they plan on using Rapid Deployment method in their network. NAT444 allows customers to run IPv4 services after the exhaustion of IPv4 addresses. However, this is not a viable long-term solution. Additionally, the implementation of NAT 444 will require investment in a NAT logging infrastructure.

Jivika Govil, Jivesh Govil, Navkeerat Kaur, and Harkeerat Kaur (2008) [4] have proposed an examination of IPv4 and IPv6 networks by analysing various constraints and transition mechanisms implemented in them. As IPv6 is not backward compatible with IPv4, and IPv4 cannot deal directly with IPv6 traffic there will be some amount of difficulties while dealing with address allocation and routing. Also it is not possible to move the entire internet over to IPv6 within a single day. Due to this reason several transitions mechanisms have been developed that can be used to make the IPv4 to IPv6 transition smooth. Various techniques such as Dual Stack Application Level Gateway (Dual Stack ALG), Network Address Translation - Protocol Translator (NAT-PT), Stateless IP/ICMP Translator (SIIT), Dual IP Stacks, Stateless IP/ICMP 
Translator (SIIT), Network Address Port Translation - Protocol Translation (NAPT-PT) and Tunneling have been discussed and their strengths and weaknesses have been analysed.

Table1: Methods used for IPv4 to IPv6 transition.

\begin{tabular}{|c|c|c|}
\hline Title & Methods Used & Analysis \\
\hline $\begin{array}{ll}\text { Tunnel-Based } & \text { IPv6 } \\
\text { Transition } & \end{array}$ & $\begin{array}{l}\text { Tunnel-based IPv6 } \\
\text { transition Framework using } \\
\text { IPv6 Rapid Deployment }\end{array}$ & $\begin{array}{l}\text { Rapid Deployment mechanism is } \\
\text { convenient and easy to manage. } \\
\text { However it has low flexibility. }\end{array}$ \\
\hline 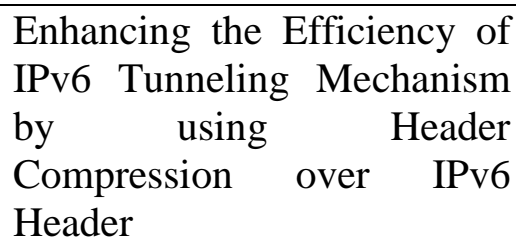 & $\begin{array}{l}\text { Header compression in } \\
\text { Tunneling mechanism }\end{array}$ & $\begin{array}{l}\text { Better deliverables are produced in } \\
\text { terms of Packet Delivery, Delay } \\
\text { and Jitter. However this technique } \\
\text { is yet to be implemented in large } \\
\text { networks }\end{array}$ \\
\hline $\begin{array}{l}\text { Simulation of IPv4-to-IPv6 } \\
\text { Dual Stack Transition } \\
\text { Mechanism (DSTM) between } \\
\text { IPv4 Hosts in Integrated } \\
\text { IPv6/IPv4 Network }\end{array}$ & $\begin{array}{l}\text { Dual Stack Transition } \\
\text { Mechanism }\end{array}$ & $\begin{array}{l}\text { DSTM is a good transition } \\
\text { mechanism, but causes more Real } \\
\text { Audio traffic consumption and } \\
\text { thereby tends to increase the } \\
\text { payload. }\end{array}$ \\
\hline $\begin{array}{l}\text { Empirical Performance of } \\
\text { IPv6 vs. IPv4 under a Dual- } \\
\text { Stack Environment }\end{array}$ & $\begin{array}{l}\text { Performance evaluation of } \\
\text { IPv6 against IPv4 in a Dual } \\
\text { Stack environment }\end{array}$ & $\begin{array}{l}\text { The analysis results indicate that } \\
\text { IPv6 provide better throughput and } \\
\text { stability. However IPv6 } \\
\text { performance of Windows-based } \\
\text { clients must be improved. }\end{array}$ \\
\hline $\begin{array}{l}\text { Comparison of Dual Stack } \\
\text { and Tunneling in Internet } \\
\text { Protocol Version } 6 \text { Transition }\end{array}$ & $\begin{array}{l}\text { Dual Stack and Tunneling } \\
\text { mechanism }\end{array}$ & $\begin{array}{l}\text { Dual Stack transition methods } \\
\text { shows better performance in the } \\
\text { network compared to Tunneling in } \\
\text { terms of Throughput and Delay } \\
\text { Jitter. }\end{array}$ \\
\hline
\end{tabular}

\section{CONCLUSIONS \& RECOMMENDATIONS}

A comprehensive study regarding the IPv4 to IPv6 transition has been made and the following conclusions can be made. While comparing the Dual Stack, Tunneling and Translation mechanisms it has been found that Dual Stack provides better efficiency in terms of throughput and UDP results. Dual Stack is capable of implementing both IPv4 and IPv6 in the same device, unlike Tunneling and also does not require an additional address translator, as while dealing with network Translation. However the cost of Dual Stack is more as it needs to support both IPv4 as well as IPv6 addresses. 
Tunneling mechanism generally tends to cause excessive load on the ISP's and is harder to implement when compared to the other two mechanisms. Translation mechanism on the other hand tends to have a lack of feasibility and also needs a separate device called the Network Address Translator (NAT) in order to perform the address translation. To improve the efficiency of Tunneling mechanism a technique for IPv6 header compression has been implemented. In this process the header size of an IPv6 packet is reduced to a large extent from around 40 bytes of IPv6 header to 6 bytes in order to provide better network deliverables. However the method has only been simulated in small networks and is yet to be implemented in larger networks.

As a future work, the Dual Stack and Tunneling mechanism can be implemented by using the routing protocols RIP and OSPF. A comparative study can be made regarding their performances and the OSPF protocol can be enhanced to provide better output. The performance can be analysed in terms of Throughput, Latency and Convergence Time. By implementing the RIP and OSPF protocols we can make it easier for devices in a network to discover better routing paths. Based on the information gathered from dynamic link state changes, we can make modifications in the network in case of any failures. On finding the best routing path, it is also possible to concurrently reduce the cost of traversal.

\section{REFERENCE}

[1] Alain Durand, Chris Metz, Jiang Dong, Peng Wu and Jianping Wu, Yiu L. Lee, Yong Cui, “Tunnel-Based IPv6 Transition”, 2013, IEEE Computer Society 1089-7801/13/\$31.00 (C) 2013 IEEE.

[2] Chris Metz, Jiangchuan Liu, Jianping Wu, Peng Wu, Yong Cui, "Transition from IPv4 to IPv6: A State-of-the-Art Survey”, 2013, IEEE Communications Surveys \& Tutorials, Vol. 15, No. 3, Third Quarter 2013.

[3] Dipti Chauhan, Sanjay Sharma, "Enhancing the Efficiency of IPv6 Tunnelling Mechanism by using Header Compression over IPv6 Header”, 2015, International Journal of Advanced Research in Computer and Communication Engineering Vol. 4, Issue 4, April 2015 Copyright to IJARCCE DOI 10.17148/IJARCCE.2015.44102 446.

[4] Harkeerat Kaur, Jivesh Govil, Jivika Govil, Navkeerat Kaur, “An Examination of IPv4 and IPv6 Networks: Constraints and Various Transition Mechanisms”, 2008, 978-14244-1884-8/08/\$25.00 @2008 IEEE.

[5] Krishna Chakraborty, Nitul Dutta, S R Biradar, "Simulation of IPv4-to-IPv6 Dual Stack Transition Mechanism (DSTM) between IPv4 Hosts in Integrated IPv6/IPv4 Network", 2009, International Conference on Computers and Devices for Communication.

[6] Man-Chiu Lai, Wee Lum Tan, Wing Cheong Lau, Yuk-Nam Law, "Empirical Performance of IPv6 vs. IPv4 under a Dual-Stack Environment”, 2008, IEEE Communications Society ICC 2008.

[7] Priya Bali, "A Detail Comprehensive Review on IPv4-to-IPv6 Transition and CoExistence Strategies”, 2015, International Journal of Advanced Research in Computer Engineering \& Technology (IJARCET) Volume 4 Issue 4, April 2015.

[8] Suwat Pattaramalai, Tachapat Taesawat, "Comparison of Dual Stack and Tunneling in Internet Protocol Version 6 Transition”, 2014. 